\title{
Erratum: Symmetry Allows for Distinguishability in Totally Destructive Many-Particle Interference [PRX Quantum 2, 020326 (2021)]
}

\author{
Julian Münzberg $\odot,{ }^{1, *}$ Christoph Dittel, ${ }^{2,3}$ Maxime Lebugle, ${ }^{4}$ Andreas Buchleitner, ${ }^{2,3}$ \\ Alexander Szameit, ${ }^{5}$ Gregor Weihs, ${ }^{1}$ and Robert Keil ${ }^{1}$ \\ ${ }^{1}$ Institut für Experimentalphysik, Universität Innsbruck, Technikerstr. 25, Innsbruck 6020, Austria \\ ${ }^{2}$ Physikalisches Institut, Albert-Ludwigs-Universität Freiburg, Hermann-Herder-Str. 3, Freiburg 79104, Germany \\ ${ }^{3}$ EUCOR Centre for Quantum Science and Quantum Computing, Albert-Ludwigs-Universität Freiburg, \\ Hermann-Herder-Str. 3, Freiburg 79104, Germany \\ ${ }^{4}$ Eulitha, Studacherstrasse 7b, Kirchdorf 5416, Switzerland \\ ${ }^{5}$ Institut für Physik, University of Rostock, Albert-Einstein-Str. 23, Rostock 18059, Germany
}

(Received 20 December 2021; published 21 January 2022)

DOI: 10.1103/PRXQuantum.3.010901

In Appendix $\mathrm{E}$ of the original paper [1], there was an error in the calculation of the simulated output probability distribution of twofold coincidences for varying indistinguishabilities $V_{A B}$ and $V_{C D}$. We calculated the output probability distribution of twofold coincidences with the method described in Ref. [2]. In this method, the partial distinguishability of the particles is treated by the Hermitian distinguishability matrix whose matrix elements $\mathcal{S}_{j k}=\left\langle\Phi_{j} \mid \Phi_{k}\right\rangle$ are the internal state overlaps of the particles with labels $j$ and $k$. We incorrectly set these matrix elements equal to the Hong-Ou-Mandel interference visibility $V_{j k}$. However, $V_{j k}=\left|\left\langle\Phi_{j} \mid \Phi_{k}\right\rangle\right|^{2}$ and thus the square of the required matrix element $\mathcal{S}_{j k}$.

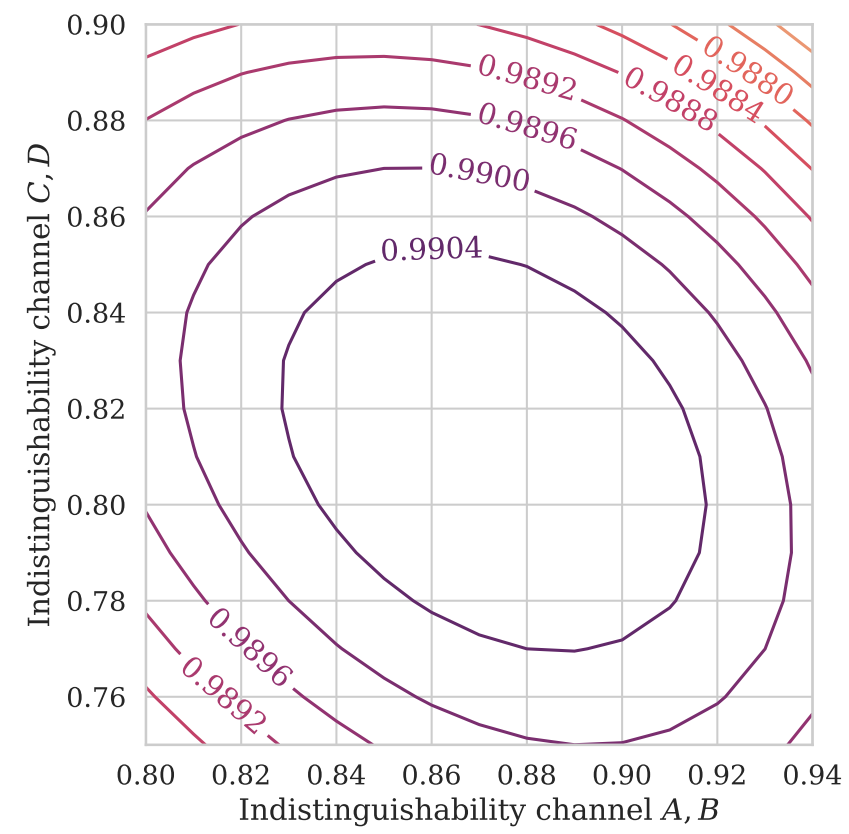

FIG. 6. The calculated fidelity for an output probability distribution of all twofold nonbunching coincidence events as a function of the indistinguishabilities $V_{A B}$ and $V_{C D}$.

*julian.muenzberg@uibk.ac.at

Published by the American Physical Society under the terms of the Creative Commons Attribution 4.0 International license. Further distribution of this work must maintain attribution to the author(s) and the published article's title, journal citation, and DOI. 
Fixing this error by setting $\mathcal{S}_{j k}=\sqrt{V_{j k}}$ results in the corrected Fig. 6 . The estimated averaged indistinguishabilities (reconstructed from the point of maximum fidelity in Fig. 6) change to $V_{A B}=0.88$ and $V_{C D}=0.81$ (before $V_{A B}=0.94$ and $V_{C D}=0.90$ ), as well as the pairwise filter offsets in the calculation of the joint spectral amplitude (JSA) to 0.91 and $1.16 \mathrm{~nm}$ between channels $A, B$ and $C, D$, respectively. The pairwise filter offsets are used to model the indistinguishability reduction by reducing the spectral overlap of the photons (see last paragraph of Appendix E in Ref. [1]). That, in turn, minimally changes the simulated results in Fig. 7 in Ref. [1], which is replaced by the new Fig. 7. These changes are not visible to the bare eye.

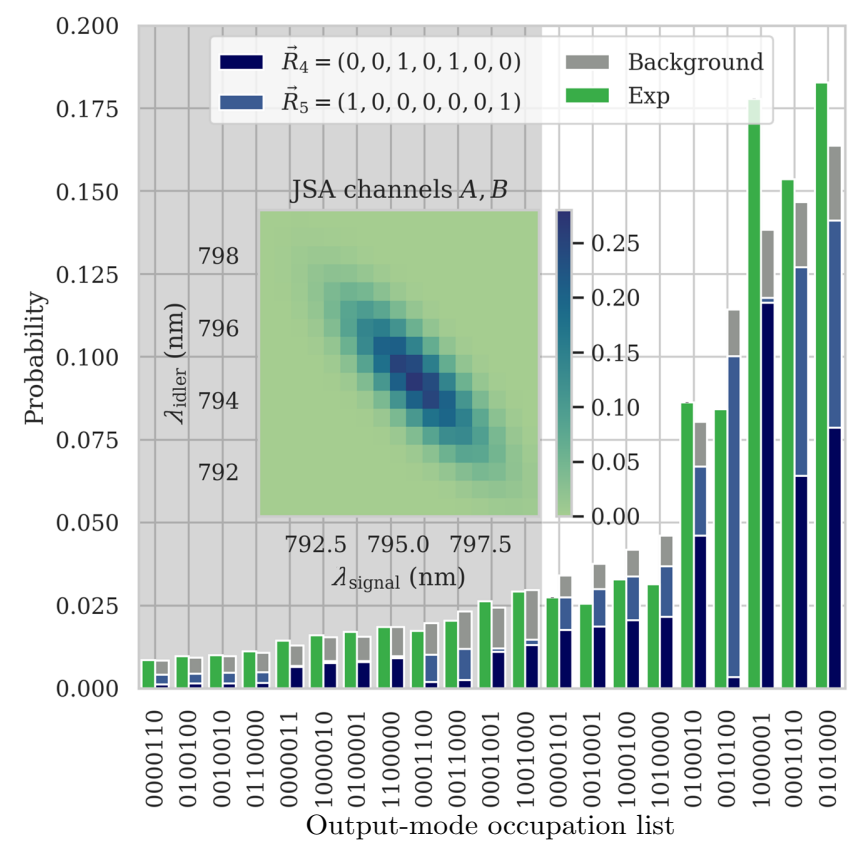

FIG. 7. The output probability distribution of all twofold nonbunching coincidence events. For the simulated result, we use optimized time-averaged indistinguishabilities of $V_{A B}=0.88$ and $V_{C D}=0.81$. The fidelity is $F=0.991$. The experimental and simulated degree of suppression violation are $D_{\exp }=0.198$ and $D_{\text {sim }}=0.197$, respectively. The experimental error bars from the counting statistics are too small to be visible. The inset shows the discretized JSA of channels $A$ and $B$ (the JSA of channels $C$ and $D$ is similar).

Successively, the simulated results in Figs. 3(a) and 3(b) in Ref. [1] change, which results in the updated Fig. 3. Table I in Ref. [1] is replaced by Table I. After correcting the error, the measured and simulated values of the degree of suppression violation agree much better with each other. For the intercycle distinguishable scenario, the simulated degree of suppression violation is within one standard deviation of the experimental results, for the mutually indistinguishable case within two standard deviations. The experimental results and the conclusions of Ref. [1] are not altered.

TABLE I. For the four investigated scenarios illustrated in Fig. 1(a) in Ref. [1], the measured $\left(\mathcal{D}_{\text {exp }}\right)$ and simulated $\left(\mathcal{D}_{\text {th }}\right)$ degree of suppression violation is listed, together with the total number $N_{\text {total }}$ of recorded four-photon coincidences.

\begin{tabular}{llll}
\hline \hline & $N_{\text {total }}$ & $\mathcal{D}_{\text {exp }}$ & $\mathcal{D}_{\text {th }}$ \\
\hline Mutually indistinguishable & 862 & $0.300 \pm 0.019$ & 0.272 \\
Intercycle distinguishable & 566 & $0.274 \pm 0.022$ & 0.284 \\
Intracycle distinguishable & 921 & $0.408 \pm 0.021$ & 0.414 \\
Mutually distinguishable & 1154 & $0.403 \pm 0.019$ & 0.415 \\
\hline \hline
\end{tabular}



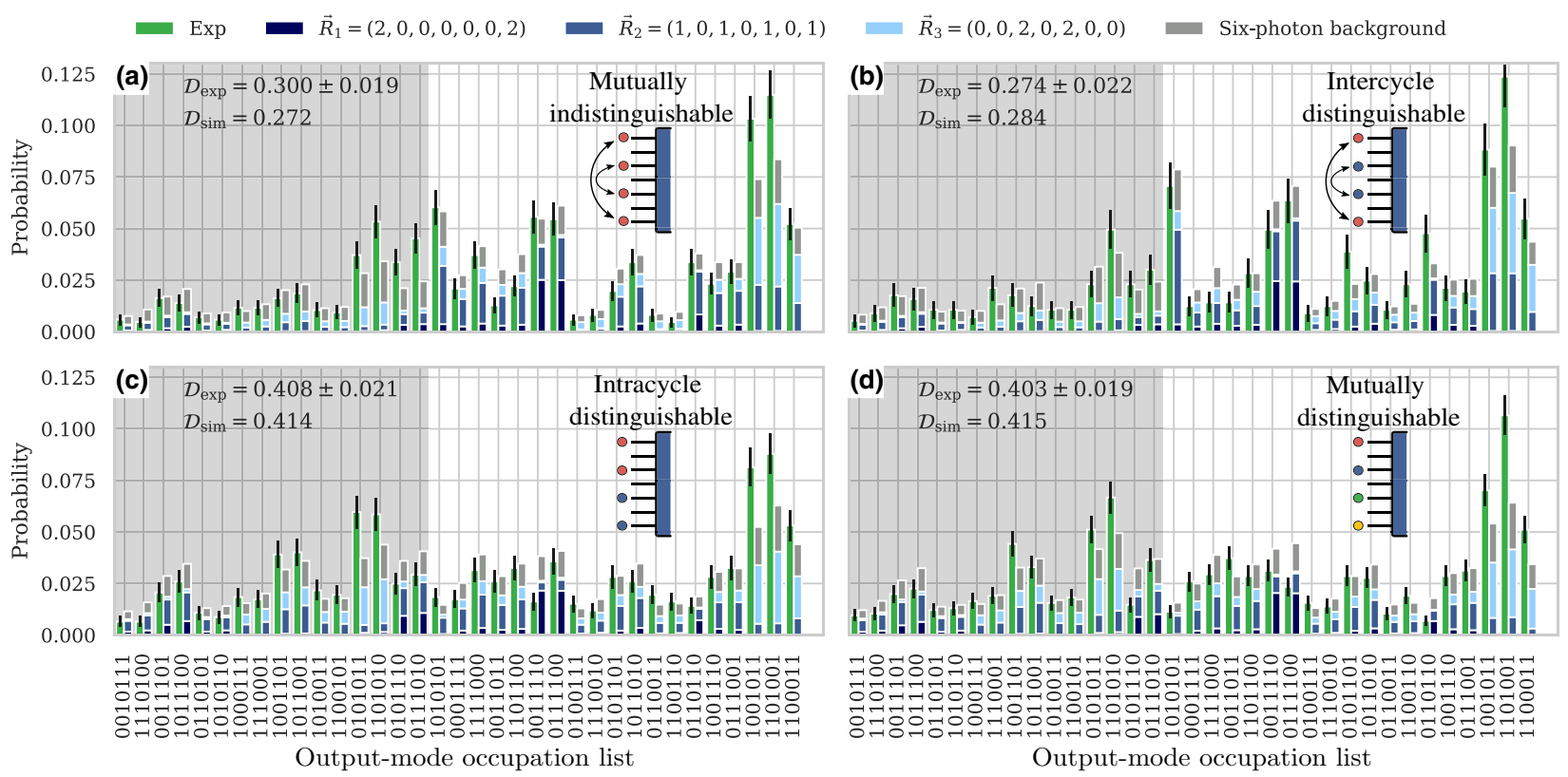

FIG. 3. The experimental and simulated output statistics of all fourfold coincidences between distinct output modes. (a),(b) The output statistics obtained for a mutually indistinguishable and for an intercycle distinguishable input state, respectively, for which all output events in the gray shaded area are ideally suppressed. (c),(d) The output statistics obtained for an intracycle distinguishable and for a mutually distinguishable input state, respectively. Here, no suppression effect is expected, even in the ideal case. The green bars correspond to experimental (Exp) data and the error bars indicate one standard deviation of the Poissonian counting statistics. The bars to the right of the experimental data correspond to a simulation of the experiment, with the dark blue, blue, light blue, and gray parts of the bars indicating the contribution stemming from the input-mode occupation $\vec{R}_{1}, \vec{R}_{2}, \vec{R}_{3}$ [see Eq. (5) in Ref. [1]] and from the six-photon background of the photon source, respectively.

[1] J. Münzberg, C. Dittel, M. Lebugle, A. Buchleitner, A. Szameit, G. Weihs, and R. Keil, Symmetry allows for distinguishability in totally destructive many-particle interference, PRX Quantum 2, 020326 (2021).

[2] M. C. Tichy, Sampling of partially distinguishable bosons and the relation to the multidimensional permanent, Phys. Rev. A 91, $022316(2015)$. 